OPEN ACCESS

Edited by:

Tommaso Pizzorusso,

University of Florence, Italy

Reviewed by:

Esther Udina,

Autonomous University of Barcelona,

Spain

Melissa R. Andrews, University of Southampton,

United Kingdom

*Correspondence:

Barbara Hausott

barbara.hausott@i-med.ac.at

Received: 02 October 2019

Accepted: 20 December 2019

Published: 21 January 2020

Citation:

Jamsuwan S, Klimaschewski L and

Hausott B (2020) Simultaneous

Knockdown of Sprouty2 and PTEN Promotes Axon Elongation of Adult

Sensory Neurons.

Front. Cell. Neurosci. 13:583.

doi: 10.3389/fncel.2019.00583

\section{Simultaneous Knockdown of Sprouty2 and PTEN Promotes Axon Elongation of Adult Sensory Neurons}

\author{
Sataporn Jamsuwan, Lars Klimaschewski and Barbara Hausott* \\ Institute of Neuroanatomy, Medical University of Innsbruck, Innsbruck, Austria
}

Sprouty2 (Spry2) and phosphatase and tensin homolog deleted on chromosome 10 (PTEN) are both well-established regulators of receptor tyrosine kinase (RTK) signaling, and knockdown of Spry2 or PTEN enhances axon regeneration of dorsal root ganglia (DRG) neurons. The major role of Spry2 is the inhibition of the rat sarcoma RAS/extracellular signal-regulated kinase (ERK) pathway, whereas PTEN acts mainly as an inhibitor of the phosphoinositide 3-kinase (PI3K)/Akt pathway. In non-neuronal cells, Spry2 increases the expression and activity of PTEN, and PTEN enhances the amount of Spry2 by the inhibition of the microRNA-21 (miR-21) that downregulates Spry2. Applying dissociated DRG neuron cultures from wild-type (WT) or Spry2 deficient mice, we demonstrate that PTEN protein was reduced after $72 \mathrm{~h}$ during rapid axonal outgrowth on the laminin substrate. Furthermore, PTEN protein was decreased in DRG cultures obtained from homozygous Spry2-/- knockout mice. Vice versa, Spry2 protein was reduced by PTEN siRNA in WT and heterozygous Spry2+/- neurons. Knockdown of PTEN in DRG cultures obtained from homozygous Spry2-/- knockout mice promoted axon elongation without increasing axonal branching. Activation of Akt, but not ERK, was stronger in response to PTEN knockdown in homozygous Spry2-/- DRG neurons than in WT neurons. Together, our study confirms the important role of the signaling modulators Spry2 and PTEN in axon growth of adult DRG neurons. Both function as endogenous inhibitors of neuronal growth factor signaling and their simultaneous knockdown promotes axon elongation more efficiently than the single knockdown of each inhibitor. Furthermore, Spry2 and PTEN are reciprocally downregulated in adult DRG neuron cultures. Axon growth is influenced by multiple factors and our results demonstrate that the endogenous inhibitors of axon growth, Spry2 and PTEN, are co-regulated in adult DRG neuron cultures. Together, our data demonstrate that combined approaches may be more useful to improve nerve regeneration than targeting one single inhibitor of axon growth.

Keywords: Sprouty2 (Spry2), PTEN (phosphatase and tensin homolog deleted on chromosome 10), DRG neurons, axon regeneration, pAkt (phosphorylated Akt) 


\section{INTRODUCTION}

Peripheral nerves are provided with the ability to regenerate in response to injuries but recovery after peripheral nerve injury is still highly limited and functional outcomes are often poor (Skouras et al., 2011; Klimaschewski et al., 2013). Successful regeneration is dependent on extrinsic factors in the environment as well as on the intrinsic regenerative capabilities of neurons. One reason for the limitations in peripheral nerve regeneration is the presence of intracellular inhibitors of neuronal growth factor signaling (Duraikannu et al., 2019). Among them are Sprouty2 (Spry2) and the dual-specificity phosphatase and tensin homolog deleted on chromosome 10 (PTEN), which act as key regulators of receptor tyrosine kinase (RTK) signaling. The assigned major role of Spry2 is the inhibition of the rat sarcoma (RAS)/extracellular signalregulated kinase (ERK) pathway (Mason et al., 2006; Hausott and Klimaschewski, 2019b), whereas PTEN has mainly been described as an inhibitor of the phosphoinositide 3-kinase (PI3K)/Akt pathway (Vazquez and Sellers, 2000; Krishnan et al., 2016). Both, the Ras/ERK and the PI3K/Akt pathway, are key players in axon growth of dorsal root ganglia (DRG) neurons (Chan et al., 2014; Hausott and Klimaschewski, 2019a).

Spry2 is highly expressed by adult DRG neurons and downregulation of Spry2 by shRNAs promotes axon growth of adult DRG neurons in vitro whereas overexpression of Spry2 inhibits axon growth (Hausott et al., 2009). DRG cultures from Spry2 knockout mice reveal enhanced axon elongation of heterozygous Spry2+/- neurons, whereas homozygous Spry2-/- neurons exhibit an axonal branching phenotype. In vivo studies with heterozygous Spry $2+1-$ mice confirmed a better recovery following sciatic nerve crush and increased levels of GAP-43, a downstream target of ERK signaling (Marvaldi et al., 2015). Although Spry2 mRNA was not altered in response to a sciatic nerve lesion in our previous study, microRNA-21 (miR-21) is upregulated in the DRG after a peripheral nerve transection and reduces Spry2 protein levels in DRG cultures. Together, these studies confirm the important role of Spry2 in nerve regeneration (Hausott et al., 2009; Strickland et al., 2011).

PTEN is present in the intact and injured adult DRG with particularly high expression in small-diameter nociceptive neurons that bind isolectin B4 (IB4). Downregulation of PTEN increases axon growth of adult DRG neurons in vitro and this effect is even stronger in pre-lesioned neurons that were axotomized before the preparation of the culture. Furthermore, in vivo knockdown of PTEN promotes regeneration in response to a sciatic nerve transection. The effect of PTEN inhibition on axon growth of adult DRG neurons is independent of mammalian target of rapamycin (mTOR), whereas the same effect on axon growth of motor neurons is dependent on mTOR (Christie et al., 2010; Ning et al., 2010). PTEN is downregulated by miR-222 or by the ubiquitin ligase neural precursor cell expressed developmentally down-regulated protein 4 (NEDD4) in DRG neurons. MiR-222 is upregulated following sciatic nerve transection and promotes neurite outgrowth of adult DRG neurons, whereas knockdown of NEDD4 decreases axon growth of DRG neurons through upregulation of PTEN (Christie et al.,
2012; Zhou et al., 2012). Although miR-21 downregulates PTEN in different cell types including neurons, it has no effect on PTEN protein levels in DRG neurons (Krichevsky and Gabriely, 2009; Strickland et al., 2011; Han et al., 2014).

Since individual downregulation of Spry2 or PTEN promotes axon regeneration and Spry2 interacts with PTEN in other cell types (Masoumi-Moghaddam et al., 2014), it was the aim of the current study to investigate the effects of simultaneous knockdown of Spry2 and PTEN on axon growth of adult DRG neurons in vitro. Knockdown of PTEN by siRNAs in DRG neurons obtained from homozygous Spry2-/- knockout mice promoted axon elongation and enhanced activation of Akt in comparison to each interference alone. Furthermore, Spry2 protein levels were decreased by PTEN knockdown and PTEN protein levels were lower in Spry2 deficient neurons. These findings demonstrate a beneficial effect of simultaneous knockdown of Spry2 and PTEN on axon growth and indicate an interrelated regulation of Spry2 and PTEN in DRG neurons.

\section{MATERIALS AND METHODS}

\section{Dissociated DRG Neuron Cultures and siRNA Transfection}

Spry2-delta-ORF (Spry2 $2^{\text {tm1.1Mrt }} / \mathrm{Mmnc}$ ) knockout mice were obtained from MMRRC (Mutant Mouse Regional Resource Centre, University of North Carolina at Chapel Hill, Chapel Hill, NC, USA) and backcrossed on a 129 S1/SVImJ background as previously described (Marvaldi et al., 2015). DRG were harvested from 5 to 6 weeks old wild-type (WT), heterozygous Spry2+/- and homozygous Spry2-/- knockout mice. After removal of connective tissue, DRG were treated with Liberase ${ }^{\mathrm{TM}}$ $\mathrm{DL}$ research-grade $(9 \mathrm{mg} / 100 \mathrm{ml}$ in DMEM, Roche) two times for $30 \mathrm{~min}$ and with Trypsin-EDTA (Invitrogen) for 15 min. DRG were then washed and mechanically dissociated with a fire-polished Pasteur pipette in TNB 100 medium supplemented with protein-lipid complex (Biochrom), L-glutamine (Invitrogen), penicillin G sodium and streptomycinsulfate (Invitrogen). Dissociated ganglia were then centrifuged at $500 \mathrm{rpm}$ for $10 \mathrm{~min}$ through a $3.5 \%$ BSA gradient (Sigma Aldrich) to reduce the number of non-neuronal cells and debris. The pellet was washed in TNB 100 medium and centrifuged for $5 \mathrm{~min}$ at $760 \mathrm{rpm}$. Neurons were then resuspended and plated on glass coverslips or Nunc ${ }^{\mathrm{TM}}$ cell-culture treated multi dishes (24 well) pre-coated with $0.01 \%$ poly-L-lysine (Sigma Aldrich) and $10 \mu \mathrm{g} / \mathrm{ml}$ laminin (Sigma Aldrich). Neurons were cultivated in TNB 100 medium (Marvaldi et al., 2015) at $37^{\circ} \mathrm{C}$ with $10 \% \mathrm{CO}_{2}$ according to the manufacturer's instructions. PTEN knockdown was induced using the Accell siRNA delivery system (Dharmacon) designed to optimize transfection of primary cells. Transfection efficiency of Accell siRNA was assessed by imaging the uptake of Accell green non-targeting siRNA (D-001950-01). The target sequences of the Accell mouse PTEN SMARTpool siRNA mixture (siPTEN) were as follows: 5'-UGAUGAUGU AGUAAGGUUU-3', 5'-GCGCUAUGUAUAUUAUUAU-3', $5^{\prime}$-GUAGUAGGCUCAAAUAUAC- $3^{\prime}$ and $5^{\prime}$-GUUACAAGU UACAUGUUUA-3'. Accell non-targeting siRNA pool (siNT; 
D-001910-10) was used as a control. After attachment of neurons after $2 \mathrm{~h}$, they were transfected with $1 \mu \mathrm{M}$ of Accell siRNA in Accell siRNA delivery media (Dharmacon) according to the manufacturer's instructions (Ning et al., 2012). Neurons were used for experiments $24 \mathrm{~h}, 48 \mathrm{~h}$ or $72 \mathrm{~h}$ after transfection.

\section{Quantitative Real-Time PCR (qPCR)}

To evaluate siRNA-induced knockdown of PTEN mRNA in DRG neuron cultures, RNA was collected $24 \mathrm{~h}, 48 \mathrm{~h}$ and $72 \mathrm{~h}$ after transfection using the ExCellenCT Lysis Kit (ABM) according to the manufacturer's protocol. To determine endogenous PTEN mRNA in DRG tissue, lumbar DRG (L3-L5) were extracted, frozen immediately in liquid nitrogen and RNA was isolated using TRI Reagent RT (Molecular Research Center). RNA template was used for cDNA synthesis with iScript ${ }^{\mathrm{TM}}$ cDNA Synthesis Kit (Bio-Rad). Quantitative real-time PCR (qPCR) was performed with the CFX Connect ${ }^{\mathrm{TM}}$ Real-Time PCR Detection System (Bio-Rad) in a final volume of $20 \mu \mathrm{l}$ with SsoAdvanced ${ }^{\mathrm{TM}}$ Universal SYBR ${ }^{\circledR}$ Green Supermix (BioRad) and QuantiTect ${ }^{\circledR}$ primer assays (Qiagen) for PTEN (Mm_Pten_1_SG, QT00141568) and HPRT1 (Mm_Hprt_1_SG, QT00166768). The qPCR reactions were performed in 40 cycles with $15 \mathrm{~s}$ at $95^{\circ} \mathrm{C}$ and $30 \mathrm{~s}$ at $60^{\circ} \mathrm{C}$.

\section{Immunocytochemistry and Axon Growth Analysis}

DRG neuron cultures were fixed for 15 min with $4 \%$ buffered paraformaldehyde (PFA) at RT, permeabilized with $0.5 \%$ Triton X-100 for 15 min and blocked with $10 \%$ goat serum for $1 \mathrm{~h}$. For axon growth analysis, neurons were pre-fixed with $1 \%$ PFA and 5\% sucrose for $10 \mathrm{~min}$ before fixation to prevent the breakage of axons. Primary antibodies (Cell Signaling Technology, Danvers, MA, USA: anti-PTEN, \#9188, 1:250; R\&D systems, Minneapolis, MN, USA: anti-TuJ-1 neuron-specific $\beta$-III tubulin, MAB1195, 1:1,000) or isolectin GS-IB4 Alexa Fluor 488 (1:500, Invitrogen) diluted in $0.3 \%$ BSA was applied overnight at $4^{\circ} \mathrm{C}$. After washing with PBS three times, secondary antibody (goat anti-rabbit Alexa Fluor 546, 1:1,000; Invitrogen) was added for $90 \mathrm{~min}$ at room temperature. No unspecific binding of secondary antibody was observed. Nuclei were stained with Hoechst (10 min, 1:20,000; Invitrogen) and coverslips were mounted in fluorescence mounting medium (Dako Omnis). Fluorescently labeled neuronal cell bodies and axons were imaged using an inverted fluorescence microscope (Zeiss AxioObserver Z1) equipped with a SPOT insight QE camera and VisiView ${ }^{\circledR}$ imaging software. The fluorescence intensity of PTEN immunostaining of cell bodies was evaluated after background subtraction applying MetaMorph ${ }^{\circledR}$ morphometry software. For axon growth analysis, the maximal distance of the longest axon, the number of branch points per neuron and the total axonal length were quantified using MetaMorph ${ }^{\circledR}$ morphometry software. If necessary, axon images were stitched before analysis using Fiji (ImageJ win64) program. All morphologically intact neurons per coverslip with axons longer than two cell body diameters were analyzed. Branches that were longer than the cell body diameter were counted as branch points.

\section{Western Blotting}

Total cell lysates from DRG cultures were prepared with RIPA II buffer supplemented with $20 \mu \mathrm{g} / \mathrm{ml}$ complete protease inhibitor cocktail (Roche Diagnostics) and phosphatase inhibitor cocktail II and III (1:100, Sigma Aldrich) followed by sonication. Protein concentrations were determined using Bradford protein assay reagent (Bio-Rad). Ten microgram of protein were separated by sodium dodecyl sulfate-polyacrylamide gel electrophoresis (SDSPAGE) and transferred to Immobilon-FL-PVDF membrane (Millipore). Membranes were blocked with Odyssey ${ }^{\circledR}$ blocking buffer (LI-COR Biosciences, Lincoln, NE, USA) in phosphatebuffered saline (PBS) and incubated with primary antibodies (Cell Signaling Technology, Danvers, MA, USA: anti-PTEN, \#9188, 1:1,000; anti-pAkt, \#4060, 1:2,000; anti-Akt, \#2920, 1:2,000; anti-pERK, \#9101, 1:1,000; anti-ERK, \#9107, 1:2,000; anti-GAPDH, \#5174, 1:1,000; Abcam: anti-Sprouty2, \#60719, 1:500) overnight at $4^{\circ} \mathrm{C}$. Secondary fluorescently labeled antibodies (LI-COR Biosciences, Lincoln, NE, USA: IRDye ${ }^{\circledR}$ 680RD goat anti-mouse and IRDye ${ }^{\circledR} 800 \mathrm{CW}$ goat anti-rabbit, $1: 20,000)$ were detected by the Odyssey FC Imaging System (LICOR Biosciences, Lincoln, NE, USA). The band intensities were quantified after background subtraction using Image Studio Lite Software version 5.2 (LI-COR Biosciences, Lincoln, NE, USA).

\section{Statistical Analysis}

Data presented as bar graphs are mean values \pm standard error of the mean (SEM) or standard deviation (SD) of at least three independent experiments. Statistical analysis was performed using one-way ANOVA followed by Bonferroni's post hoc test. Differences with a $p<0.05$ were considered statistically significant $\left({ }^{*} p<0.05,{ }^{* *} p<0.01,{ }^{* * *} p<0.001\right.$ or $* * * * p<0.0001)$.

\section{RESULTS}

\section{Endogenous PTEN Levels Are Reduced in Culture}

In DRG tissue, PTEN is highly expressed by the lectin IB4-positive population of small neurons (Christie et al., 2010). Thus, we first investigated the distribution of PTEN in DRG subpopulations after $2 \mathrm{~h}, 24 \mathrm{~h}$, and $72 \mathrm{~h}$ in culture. The PTEN immunoreactivity was significantly higher $2 \mathrm{~h}$ after plating in IB4-positive neurons reflecting the in vivo situation. However, immunofluorescence of PTEN decreased in a time-dependent manner in all DRG neuron populations during in vitro cultivation, and the difference between the IB4-positive and IB4-negative DRG subpopulation was strongly reduced after $72 \mathrm{~h}$ (Figures 1A,B). Glial cells present in DRG cultures were distinguished by their typical morphology of small nuclei that are surrounded by a narrow cytoplasmic margin. These associated glial cells revealed much weaker PTEN immunoreactivity as compared to DRG neurons (Figure 1C).

\section{PTEN Knockdown by Accell siRNA}

Methods to introduce RNA interference into adult DRG neurons are still challenging. We used the Accell siRNA technology from Dharmacon which consists of chemically modified siRNAs that 

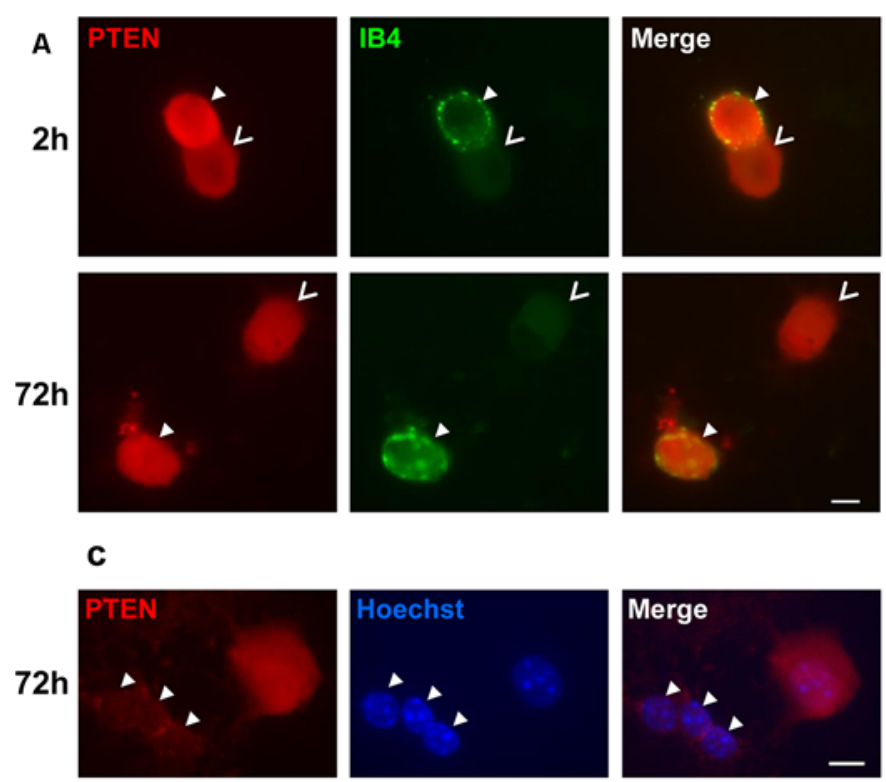

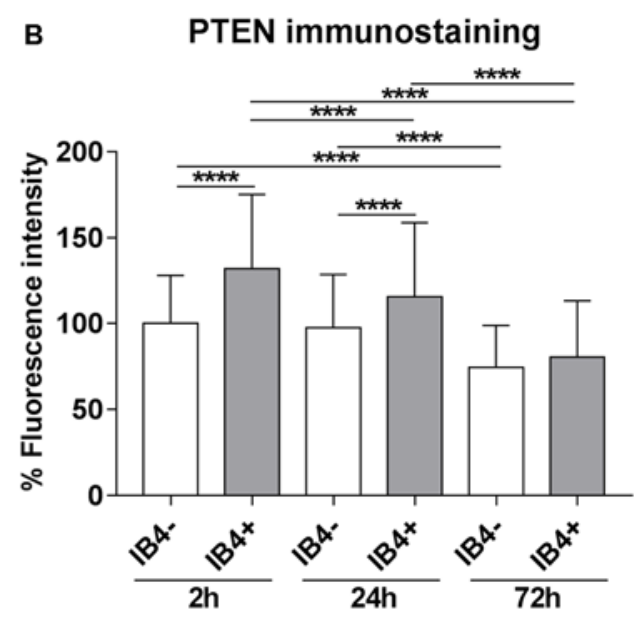

FIGURE 1 | PTEN immunofluorescence in dorsal root ganglia (DRG) neuron subpopulations and glial cells. (A) Examples of PTEN immunoreactivity in IB4-positive (IB4+, filled arrowheads) and IB4-negative (IB4-, open arrowheads) DRG neurons. Bar = $10 \mu \mathrm{m}$. (B) PTEN fluorescence intensity was significantly higher in IB4-positive neurons after $2 \mathrm{~h}$ but this difference decreased in a time-dependent manner during cultivation. ${ }^{* * * *} p<0.0001$ for the indicated comparisons, mean \pm standard deviation (SD) of three independent experiments with a total number of neurons per group $>180$. (C) Immunoreactivity against PTEN is reduced in glial cells (arrowheads) compared to neurons. Bar $=10 \mu \mathrm{m}$.

are optimized for primary cells. First of all, the transfection efficiency of Accell siRNA was assessed by imaging the uptake of Accell green non-targeting siRNA into DRG neurons. Accell green control siRNAs were taken up by $95.0 \%( \pm 11.0)$ of the neurons that were immunoreactive to $\beta$-III tubulin after $72 \mathrm{~h}$ in culture (Figure 2A). Next, we assessed the downregulation of PTEN by the Accell siRNA using qPCR. PTEN siRNA reduced mRNA expression after $24 \mathrm{~h}, 48 \mathrm{~h}$ and $72 \mathrm{~h}$ with the strongest downregulation at $72 \mathrm{~h}$ (by $68.4 \%$ compared to the non-targeting siRNA, Figure 2B). To confirm the effect of PTEN siRNA on protein levels, immunoreactivity against PTEN was measured after $24 \mathrm{~h}, 48 \mathrm{~h}$ and $72 \mathrm{~h}$. PTEN siRNA reduced PTEN immunoreactivity in a time-dependent manner with the strongest reduction by almost 50\% compared to non-targeting siRNA at $72 \mathrm{~h}$ (Figure 2C). Immunoreactivity against PTEN was again reduced in DRG neurons transfected with non-targeting siRNA at $48 \mathrm{~h}$ and $72 \mathrm{~h}$ in culture although the reduction in mRNA was not statistically significant (Figures 2B,C). Since the strongest mRNA and protein knockdown induced by the PTEN siRNA was observed after $72 \mathrm{~h}$, further siRNA experiments were performed at this time point.

\section{Spry2 and PTEN Protein Levels Are Co-regulated in DRG Neurons}

Knockdown of PTEN protein by siRNA was also confirmed by Western blotting after $72 \mathrm{~h}$ (Figure 3A). Although PTEN and Spry2 are expressed by adult DRG neurons in vitro and in vivo (Hausott et al., 2009; Christie et al., 2010; Marvaldi et al., 2015), a possible correlation between Spry2 and PTEN in DRG neurons has not been investigated so far. PTEN protein was significantly reduced in homozygous Spry2-/knockout cultures compared to the WT although no reduction in PTEN mRNA levels was observed in DRG tissue suggesting a possible post-transcriptional regulation of PTEN in response to Spry2 knockdown (Figures 3A,B). DRG cultures from heterozygous Spry2+/- mice revealed reduced Spry2 protein levels and complete knockdown of Spry2 protein was observed in homozygous Spry2-/- DRG cultures. Furthermore, PTEN siRNA markedly reduced Spry2 protein levels of WT and heterozygous Spry2+/- DRG cultures (Figure 3C). These findings imply the reciprocal regulation of Spry2 and PTEN in adult DRG neuron cultures.

\section{Simultaneous Knockdown of Spry2 and PTEN Promotes Axon Elongation}

We observed the effects of Spry2 knockdown on axon outgrowth of dissociated DRG neurons after $24 \mathrm{~h}$ in our previous study with improved axonal elongation of heterozygous Spry2+/- cultures and increased axonal branching of homozygous Spry2-/cultures (Marvaldi et al., 2015). Since optimal knockdown with Accell siRNA is usually observed after $72 \mathrm{~h}$ (according to the manufacturer's instructions) and PTEN knockdown was stronger after $72 \mathrm{~h}$ than after $24 \mathrm{~h}$ in our cultures, axon growth was analyzed after $72 \mathrm{~h}$. After $72 \mathrm{~h}$ of PTEN knockdown, the maximal distance of the longest axon (by 16.0\%) and the total axonal length (by 32.3\%) increased in WT neurons, whereas the number of branch points did not significantly change (Figures 4A,B). Neurons from heterozygous Spry2+/and homozygous Spry2-/- knockout mice revealed both a branching phenotype at $72 \mathrm{~h}$, while the maximal distance 
A
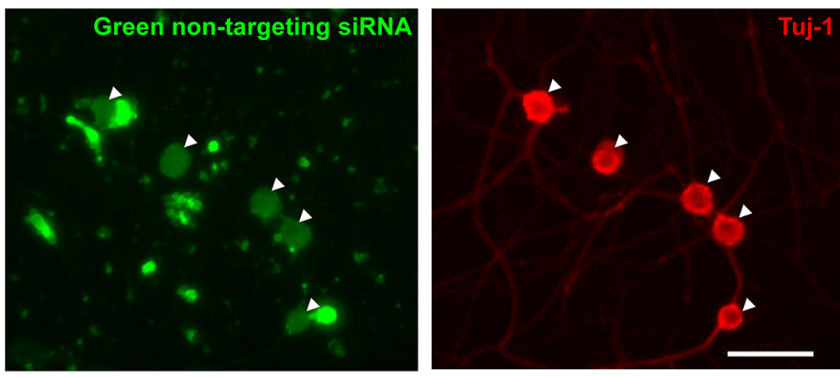

B

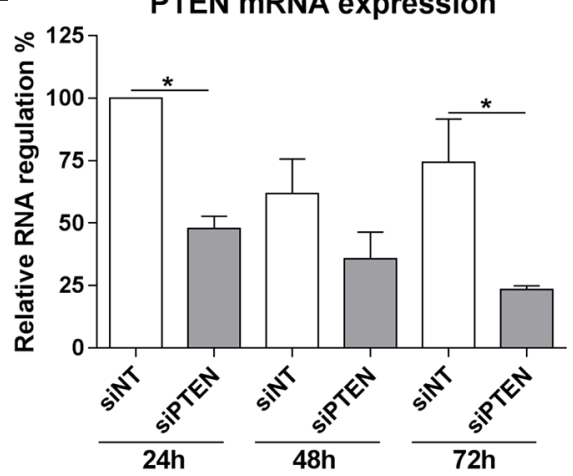

c PTEN immunostaining
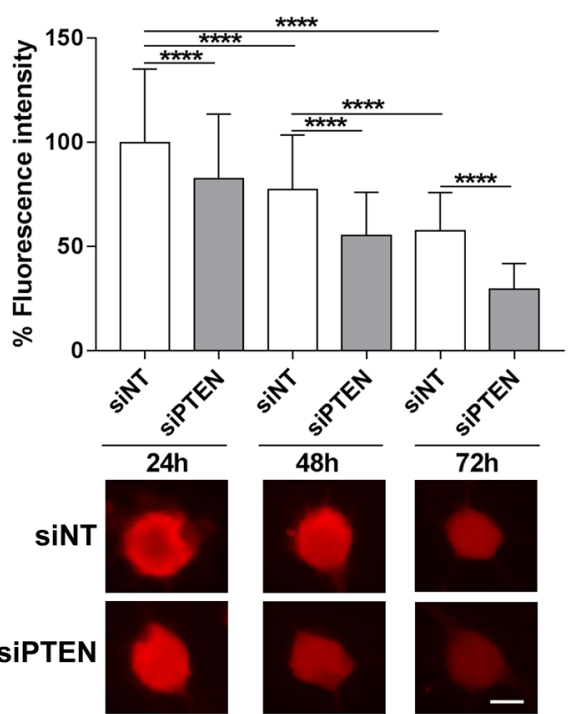

FIGURE 2 | Transfection of adult DRG cultures with Accell siRNA and knockdown with PTEN siRNA (siPTEN) compared to non-targeting siRNA (siNT). (A) Uptake of Accell green non-targeting siRNA (arrowheads) into the cell bodies of dissociated DRG neuron cultures labeled with $\beta$-III tubulin (Tuj-1, red). Bar $=50 \mu \mathrm{m}$. (B) PTEN siRNA induces downregulation of PTEN mRNA in DRG neuron cultures as determined by quantitative real-time PCR (qPCR). Reduction of PTEN mRNA was observed after $24 \mathrm{~h}, 48 \mathrm{~h}$ and $72 \mathrm{~h}$ with the strongest downregulation compared to the non-targeting siRNA at $72 \mathrm{~h}$. ${ }^{*} p<0.05$ for the indicated comparisons, mean \pm standard error of the mean (SEM) of three independent experiments. (C) Immunofluorescence measurements of PTEN following background correction indicate the reduction of endogenous PTEN protein levels in a time-dependent manner in neurons transfected with non-targeting siRNA, which is further reduced by PTEN siRNA after $24 \mathrm{~h}, 48 \mathrm{~h}$ and $72 \mathrm{~h}$ with the strongest downregulation at $72 \mathrm{~h} .{ }^{* * * *} \mathrm{p}<0.0001$ for the indicated comparisons, mean $\pm \mathrm{SD}$ of three independent experiments with a total number of neurons per group $>400$. Bar $=10 \mu \mathrm{m}$.

was not increased. However, knockdown of PTEN promoted axonal elongation by homozygous Spry2-/- deficient DRG neurons significantly stronger (by 36.0\%) than PTEN single downregulation without increasing the number of branch points. Together, simultaneous knockdown of Spry2 and PTEN promoted axon elongation stronger than PTEN single knockdown without further enhancing axon branching that is observed after a single knockdown of Spry2 after $72 \mathrm{~h}$.

\section{Simultaneous Knockdown of Spry2 and PTEN Enhances Akt Activation}

PTEN is a well-established inhibitor of Akt signaling and the described main function of Spry2 is the inhibition of the ERK pathway (Vazquez and Sellers, 2000; Mason et al., 2006). Therefore, phosphorylation of Akt and ERK in response to the simultaneous knockdown of Spry2 and PTEN in DRG cultures was analyzed by Western blotting. As demonstrated in our previous studies (Hausott et al., 2009; Marvaldi et al., 2015), immunoblotting revealed no changes in Akt phosphorylation in Spry2 deficient DRG neurons (Figure 5A). Thus, the modest reduction of PTEN protein observed in Spry2 deficient DRG neurons (Figure 3A) did not induce Akt activation. However, PTEN knockdown promoted Akt phosphorylation as expected and this effect was stronger in homozygous Spry2-/- DRG neurons than in WT neurons $(p=0.07)$. No difference in phosphorylation of ERK was observed in response to PTEN knockdown. In homozygous Spry2-/- knockout cultures, activation of ERK increased with a $p$-value of 0.07 but this effect was abolished by the PTEN siRNA (Figure 5B).

\section{DISCUSSION}

It was demonstrated before by our group and others that single knockdown of Spry2 or PTEN promotes axon growth of adult DRG neuron cultures (Hausott et al., 2009; Christie et al., 2010; Marvaldi et al., 2015). In this study, we provide for the first time evidence that simultaneous knockdown of Spry2 and PTEN has a stronger effect on axon elongation and activation of Akt than downregulation of PTEN alone. Furthermore, we demonstrate that PTEN protein levels decrease during in vitro cultivation and that Spry2 and PTEN protein levels are downregulated reciprocally in adult DRG neuron cultures.

PTEN is highly expressed by the lectin IB4-positive population of small DRG neurons 3 days after sciatic nerve lesion (Christie et al., 2010). In DRG neuron culture, PTEN immunoreactivity was significantly higher in IB4-positive neurons $2 \mathrm{~h}$ after plating reflecting the in vivo situation after lesion. During cultivation PTEN immunofluorescence 


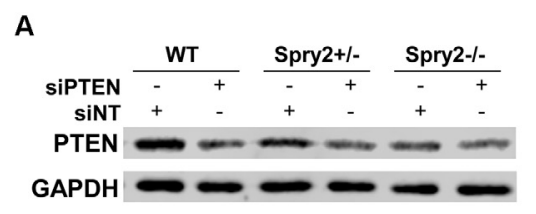

C

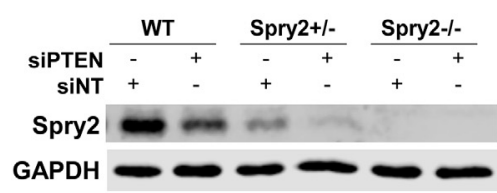

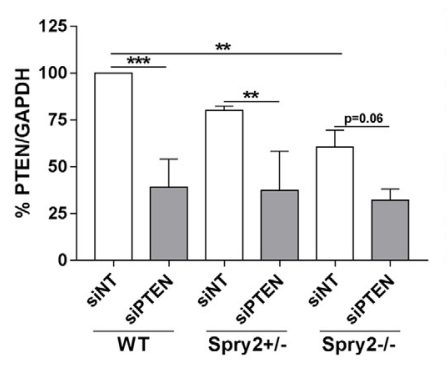
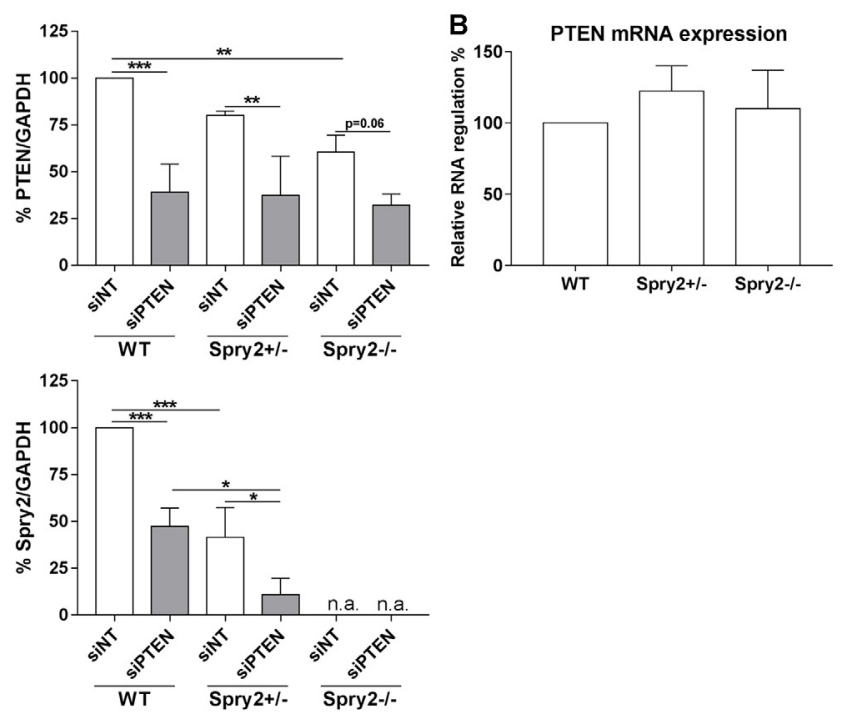

FIGURE 3 | Western blot analysis of PTEN and Spry2 $72 \mathrm{~h}$ after PTEN siRNA (siPTEN) transfection of wild-type (WT), heterozygous Spry2+/- and homozygous Spry2-/- DRG cultures compared to non-targeting siRNA (siNT). (A) PTEN protein decreased in response to PTEN siRNA in WT, heterozygous Spry2+/- and homozygous Spry2-/- DRG cultures. In addition, PTEN was reduced in homozygous Spry2-/- cultures transfected with non-targeting siRNA as compared to WT cultures. ${ }^{* *} p<0.01,{ }^{* * *} p<0.001$ for the indicated comparisons, mean $\pm \mathrm{SD}$ of three independent experiments. (B) No difference in PTEN mRNA expression was observed in DRG tissue from heterozygous Spry2+/- and homozygous Spry2-/- knockout mice compared to the WT. Mean \pm SEM of four independent experiments. (C) Spry2 protein decreased in heterozygous Spry2+/- cultures and was absent in homozygous Spry2-/- cultures (n.a., not applicable). In response to PTEN siRNA, Spry2 was markedly reduced in WT and heterozygous Spry2+/- cultures. ${ }^{*} p<0.05,{ }^{* * *} p<0.001$ for the indicated comparisons, mean \pm SD of three independent experiments.

decreased and after $72 \mathrm{~h}$ IB4-positive and IB4-negative DRG subpopulations revealed similar levels of PTEN. The reduction of PTEN during cultivation may facilitate neurite outgrowth especially of IB4-positive neurons after $72 \mathrm{~h}$, which are limited in their regenerative capacity in vitro after $24 \mathrm{~h}$ (Leclere et al., 2007). PTEN is expressed by Schwann cells within the sciatic nerve and in regenerating axons but PTEN levels in glia appear weaker than in regenerating axons (Christie et al., 2010). Glial cells present in our cultures exhibited reduced immunoreactivity as compared to DRG neurons. Together, these studies indicate that PTEN expression in cell bodies and axons of DRG neurons is higher than in glial cells and that PTEN levels decrease in neurons during the process of neurite outgrowth.

PTEN protein is reduced in homozygous Spry2-1- DRG cultures and Spry2 is reduced in WT and heterozygous Spry2+/DRG cultures by PTEN knockdown. Spry2 and PTEN are often concurrently deregulated in tumors and miRNAs or ubiquitin ligases appear to be involved in these processes (Wang et al., 2007; Patel et al., 2013; Zhang et al., 2016). PTEN negatively regulates miR-21 expression via the RNA-regulatory protein ribonuclease/angiogenin inhibitor 1 (RNH1), thereby preventing the downregulation of Spry2 by miR-21 in glioma cells (Kim et al., 2011; Kwak et al., 2011). MiR-21 is upregulated in response to a peripheral nerve lesion and downregulates Spry2, but not PTEN, in DRG neurons (Strickland et al., 2011). During the preparation of DRG cultures, neurons are axotomized as well, which may upregulate miR-21 in
DRG cultures since PTEN is downregulated in cultures as observed in our study. In tumor cells and primary embryonic fibroblasts, Spry2 increases the amount of PTEN and decreases its phosphorylation, thereby enhancing the activity of PTEN to suppress phosphorylation of Akt (Edwin et al., 2006; Feng et al., 2011). Vice versa, Spry2 knockdown enhances phosphorylation and nuclear translocation of PTEN (Patel et al., 2013). PTEN is downregulated by the miR-222, which itself increases following sciatic nerve transection and promotes neurite outgrowth by downregulation of PTEN (Zhou et al., 2012). The ubiquitin ligase NEDD4 downregulates Spry2 and PTEN, and knockdown of NEDD4 inhibits axon growth of DRG neurons (Edwin et al., 2010; Christie et al., 2012). PTEN mRNA levels were not reduced in homozygous Spry2-1- DRG tissue from uninjured animals although PTEN protein was reduced in homozygous Spry2-/- DRG cultures suggesting a possible post-transcriptional regulation of PTEN in response to Spry2 knockdown. However, cultured DRG neurons undergo a lesion process which may also change gene expression. Thus, the exact mechanisms of the reciprocal downregulation of Spry2 and PTEN protein, as well as downregulation of the latter during DRG cultivation, remain to be elucidated in future studies.

Simultaneous knockdown of Spry2 and PTEN had a more beneficial effect on elongating axon growth of adult DRG neurons than single knockdown of PTEN. We used the Accell PTEN siRNA optimized for primary cells which revealed the strongest knockdown at $72 \mathrm{~h}$ in culture. Furthermore, the effect 

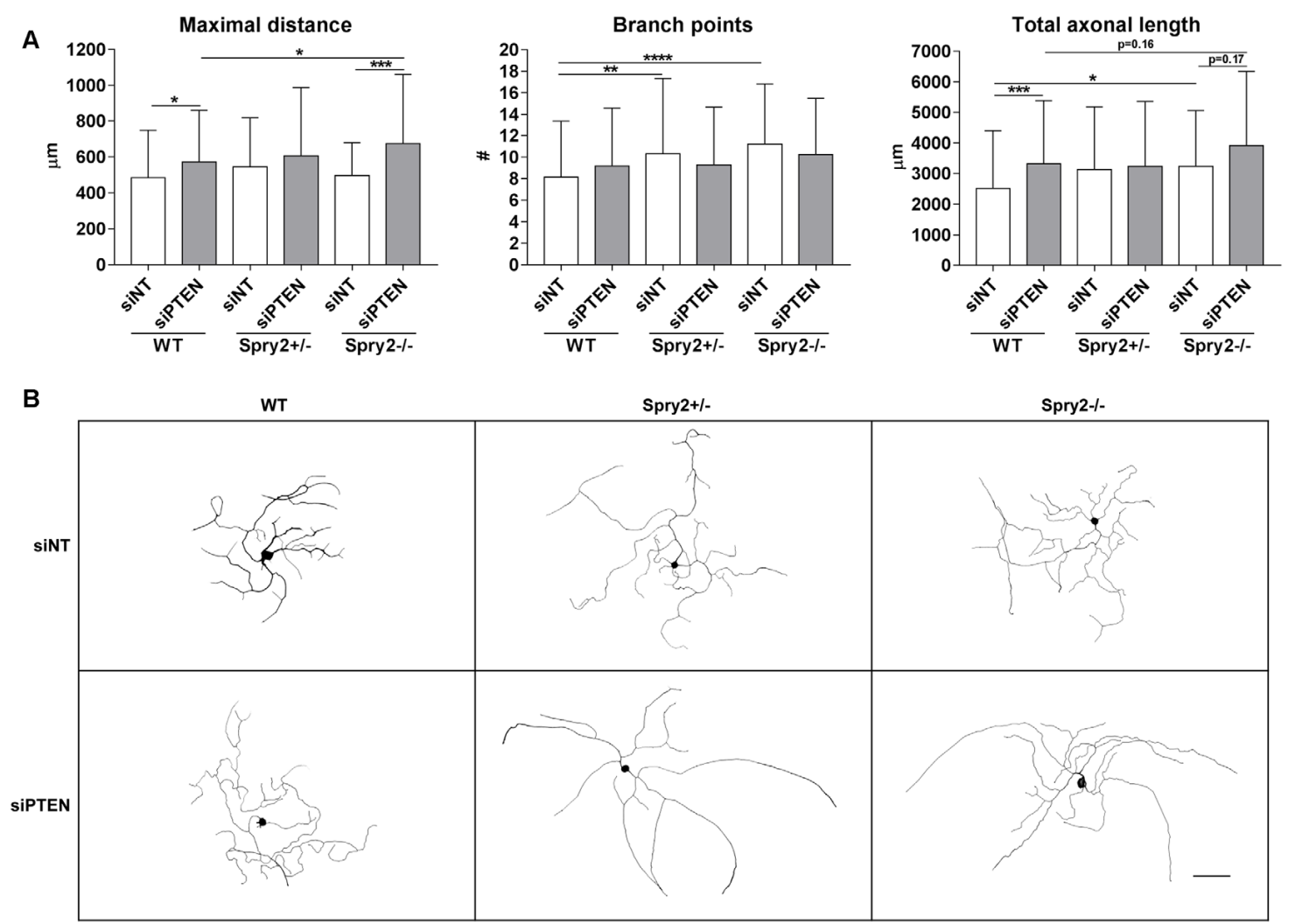

FIGURE 4 | Axon growth $72 \mathrm{~h}$ after PTEN siRNA (siPTEN) transfection of WT, heterozygous Spry2+/- and homozygous Spry2-/- DRG cultures compared to non-targeting siRNA (siNT). (A) PTEN downregulation increased the total axonal length and the length of the longest axon (maximal distance) of WT neurons without enhancement of axonal branching. Heterozygous Spry2+/- and homozygous Spry2-/- DRG cultures revealed increased branching after $72 \mathrm{~h}$. In homozygous Spry2-/- DRG neurons PTEN siRNA increased the maximal distance. ${ }^{*} p<0.05,{ }^{* *} p<0.01,{ }^{* * *} p<0.001,{ }^{* * * *} p<0.0001$ for the indicated comparisons, mean \pm SD of three independent experiments with a total number of neurons per group $>90$. (B) Representative examples of neuronal morphologies of WT, heterozygous Spry2+/- and homozygous Spry2-/- DRG cultures $72 \mathrm{~h}$ after transfection with non-targeting or PTEN siRNA. Bar $=100 \mu \mathrm{m}$.

of PTEN downregulation on axon growth of WT neurons was only visible after $72 \mathrm{~h}$ but not after $24 \mathrm{~h}$ (data not shown). Other studies using Accell siRNAs in neurons did neither observe sufficient protein knockdown nor stimulation of axon growth before $72 \mathrm{~h}$ (Ning et al., 2012; Hannila et al., 2013). In contrast to our previous study with heterozygous Spry2+/- and homozygous Spry2-/- DRG cultures which was performed after $24 \mathrm{~h}$ (Marvaldi et al., 2015), both heterozygous Spry2+/- and homozygous Spry2-/- neurons exhibited a branching phenotype after $72 \mathrm{~h}$. The PTEN siRNA did not further increase axonal branching of heterozygous Spry2+/and homozygous Spry2-l- neurons but elongative axon growth was concurrently enhanced in homozygous Spry2-1knockout cultures. Thus, knockdown of PTEN transformed the branching phenotype of homozygous Spry2-/- knockout cultures into a more elongative phenotype, which is important for successful nerve regeneration particularly at the lesion site. Other studies demonstrated as well that the combined deletion of different inhibitory signaling molecules has a better effect on axon growth than single inhibition only. Simultaneous knockdown of PTEN and suppressor of cytokine signaling 3 (SOCS3), an inhibitor of the Janus kinase/signal transducer and activator of transcription (JAK/STAT) pathway, reveals a better effect on regeneration after sciatic or optic nerve crush lesion (Sun et al., 2011; Gallaher and Steward, 2018). Although co-deletion of PTEN and SOCS3 increases sciatic nerve regrowth after lesion to a similar extent as PTEN deletion alone, simultaneous knockdown of PTEN and SOCS3 leads to more rapid recovery of thermo- and mechanosensation (Gallaher and Steward, 2018). By contrast, other studies observed that enhanced axon regrowth after lesion in response to robust mTOR activation inhibits proper innervation of the epidermis due to excessive branching (Abe et al., 2010). Our in vitro results indicate that the simultaneous knockdown of Spry2 and PTEN promotes elongative axon growth which is a precondition for successful regeneration. Furthermore, the combination of PTEN knockdown and activation of rapidly accelerated fibrosarcoma (RAF) or cyclic adenosine monophosphate (cAMP) enhances optic nerve regeneration (de Lima et al., 2012; Zhong, 2015). It is well known that axon growth is influenced by multiple inhibitory and growth-promoting factors especially in the central nervous system. Our results confirm that the development of combined approaches may be useful to improve nerve regeneration in the peripheral nervous system as well. Since the in vivo situation is 


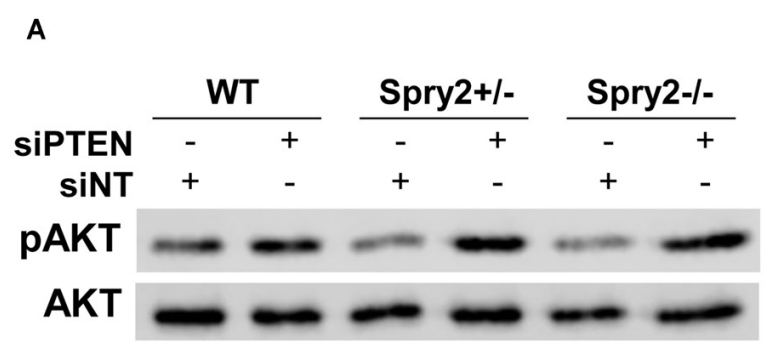

B

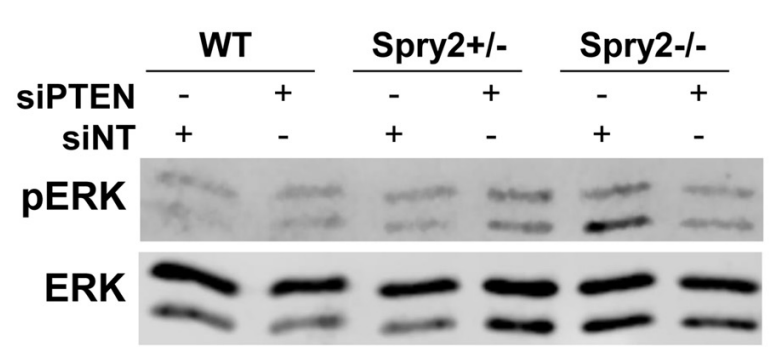

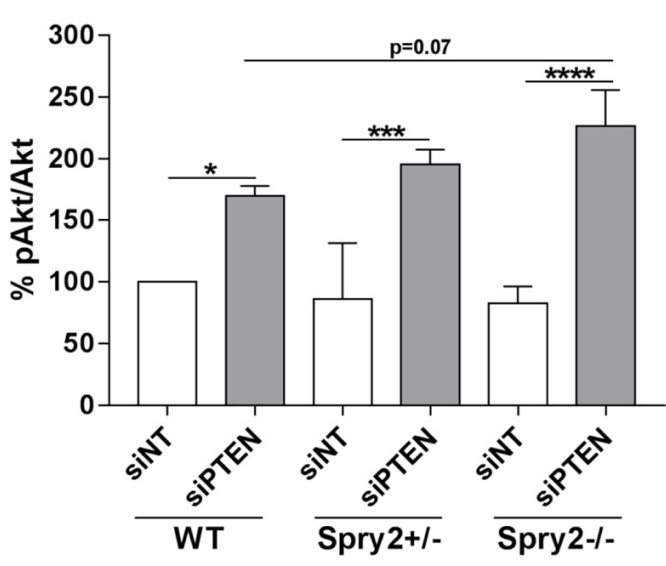

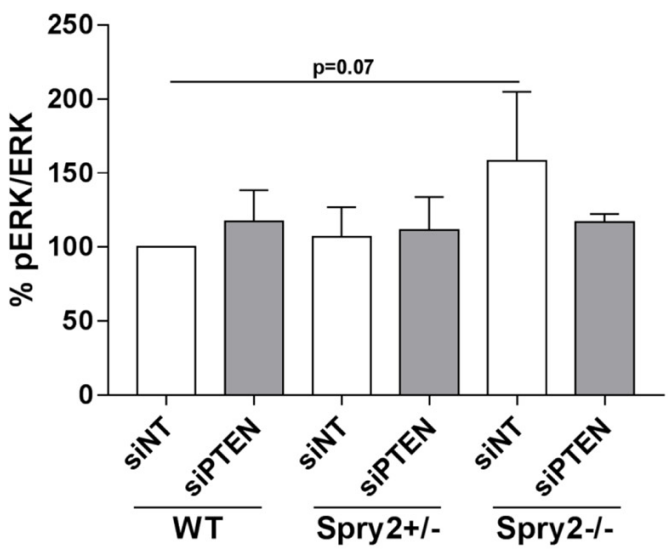

FIGURE 5 | Western blot analysis of Akt and ERK phosphorylation $72 \mathrm{~h}$ after PTEN siRNA (siPTEN) transfection of WT, heterozygous Spry2+/- and homozygous Spry2-/- DRG cultures compared to non-targeting siRNA (siNT). (A) pAkt/Akt ratios were enhanced in response to PTEN downregulation and this effect was stronger in homozygous Spry2-/- DRG cultures than in WT cultures. ${ }^{*} p<0.05,{ }^{* * *} p<0.001,{ }^{* * * *} p<0.0001$ for the indicated comparisons, mean \pm SD of three independent experiments. (B) pERK/ERK ratios were increased in homozygous Spry2-/- DRG cultures but not in response to PTEN downregulation. Mean \pm SD of three independent experiments.

much more complex in many respects, the effect of simultaneous knockdown of Spry2 and PTEN on sciatic nerve regeneration needs to be investigated in future studies.

Activation of Akt did not change in heterozygous Spry2+/and homozygous Spry2-/- neurons confirming our previous studies (Hausott et al., 2009; Marvaldi et al., 2015). Although PTEN protein was reduced in homozygous Spry2-/- cultures, pAkt was unchanged indicating that the modest reduction of PTEN by Spry 2 knockdown is not sufficient to activate detectable amounts of Akt. In response to the stronger PTEN reduction induced by the siRNA, activation of Akt was observed and this activation was stronger in homozygous Spry2-/- DRG neurons than in WT neurons, although knockdown of Spry2 alone had no effect on Akt activation. It was demonstrated before that simultaneous knockdown of Spry2 and PTEN results in higher pAkt activation than single knockdown of PTEN in prostate cancer tissue (Gao et al., 2012). The Akt pathway plays a major role in adult axon outgrowth and it is known that activation of Akt increases axonal branching in developing and adult DRG neurons (Markus et al., 2002; Jones et al., 2003). Surprisingly, our study revealed enhanced activation of pAkt by simultaneous knockdown of Spry2 and PTEN that resulted in improved axonal elongation without further increasing the branching phenotype induced by single knockdown of Spry2 after $72 \mathrm{~h}$. Furthermore, single knockdown of PTEN in WT cultures enhanced activation of Akt and axon elongation but not axonal branching after $72 \mathrm{~h}$. Activation of ERK was enhanced in homozygous Spry2-1neurons after $72 \mathrm{~h}$ as observed in our previous studies after $24 \mathrm{~h}$ (Marvaldi et al., 2015). However, knockdown of PTEN, which strongly activated Akt, diminished this effect on ERK activation. It has been shown before that strong activation of Akt inhibits the ERK pathway by serine phosphorylation of RAF or forkhead box protein O1 (FOXO1; Zimmermann and Moelling, 1999; Lee et al., 2008; Pan et al., 2017).

Taken together, our study confirms the important role of Spry2 and PTEN in axon growth of adult DRG neurons. Both are endogenous inhibitors of neuronal growth factor signaling and their simultaneous knockdown promotes axon elongation stronger than the single knockdown of each inhibitor. In vivo studies confirm the significance of the endogenous inhibitors Spry2 and PTEN that are downregulated in response to a sciatic nerve lesion by miR-21 or miR-222, respectively. Axon 
growth is influenced by multiple factors and our results confirm that combined approaches targeting different inhibitors of axon growth may be useful to improve peripheral nerve regeneration.

\section{DATA AVAILABILITY STATEMENT}

All datasets generated for this study are included in the article.

\section{ETHICS STATEMENT}

Ethical review and approval was not required for the animal study because the tissue was collected postmortem. Animal breeding was performed under the permission of the Austrian government: BMWFW-66.011/0120-WF/V/3b/2016.

\section{REFERENCES}

Abe, N., Borson, S. H., Gambello, M. J., Wang, F., and Cavalli, V. (2010). Mammalian target of rapamycin (mTOR) activation increases axonal growth capacity of injured peripheral nerves. J. Biol. Chem. 285, 28034-28043. doi: 10.1074/jbc.m110.125336

Chan, K. M., Gordon, T., Zochodne, D. W., and Power, H. A. (2014). Improving peripheral nerve regeneration: from molecular mechanisms to potential therapeutic targets. Exp. Neurol. 261, 826-835. doi: 10.1016/j.expneurol.2014. 09.006

Christie, K. J., Martinez, J. A., and Zochodne, D. W. (2012). Disruption of E3 ligase NEDD4 in peripheral neurons interrupts axon outgrowth: linkage to PTEN. Mol. Cell. Neurosci. 50, 179-192. doi: 10.1016/j.men.2012.04.006

Christie, K. J., Webber, C. A., Martinez, J. A., Singh, B., and Zochodne, D. W. (2010). PTEN inhibition to facilitate intrinsic regenerative outgrowth of adult peripheral axons. J. Neurosci. 30, 9306-9315. doi: 10.1523/JNEUROSCI.627109.2010

de Lima, S., Koriyama, Y., Kurimoto, T., Oliveira, J. T., Yin, Y., Li, Y., et al. (2012). Full-length axon regeneration in the adult mouse optic nerve and partial recovery of simple visual behaviors. Proc. Natl. Acad. Sci. U S A 109, 9149-9154. doi: 10.1073/pnas.1119449109

Duraikannu, A., Krishnan, A., Chandrasekhar, A., and Zochodne, D. W. (2019). Beyond trophic factors: exploiting the intrinsic regenerative properties of adult neurons. Front. Cell. Neurosci. 13:128. doi: 10.3389/fncel.2019.00128

Edwin, F., Anderson, K., and Patel, T. B. (2010). HECT domain-containing E3 ubiquitin ligase Nedd4 interacts with and ubiquitinates Sprouty2. J. Biol. Chem. 285, 255-264. doi: 10.1074/jbc.m109.030882

Edwin, F., Singh, R., Endersby, R., Baker, S. J., and Patel, T. B. (2006). The tumor suppressor PTEN is necessary for human Sprouty 2-mediated inhibition of cell proliferation. J. Biol. Chem. 281, 4816-4822. doi: 10.1074/jbc.m508300200

Feng, Y. H., Wu, C. L., Tsao, C. J., Chang, J. G., Lu, P. J., Yeh, K. T., et al. (2011). Deregulated expression of sprouty 2 and microRNA-21 in human colon cancer: correlation with the clinical stage of the disease. Cancer Biol. Ther. 11, 111-121. doi: 10.4161/cbt.11.1.13965

Gallaher, Z. R., and Steward, O. (2018). Modest enhancement of sensory axon regeneration in the sciatic nerve with conditional co-deletion of PTEN and SOCS3 in the dorsal root ganglia of adult mice. Exp. Neurol. 303, 120-133. doi: 10.1016/j.expneurol.2018.02.012

Gao, M., Patel, R., Ahmad, I., Fleming, J., Edwards, J., McCracken, S., et al. (2012). SPRY2 loss enhances ErbB trafficking and PI3K/AKT signalling to drive human and mouse prostate carcinogenesis. EMBO Mol. Med. 4, 776-790. doi: $10.1002 / \mathrm{emmm} .201100944$

Han, Z., Chen, F., Ge, X., Tan, J., Lei, P., and Zhang, J. (2014). miR-21 alleviated apoptosis of cortical neurons through promoting PTEN-Akt signaling pathway in vitro after experimental traumatic brain injury. Brain Res. 1582, 12-20. doi: 10.1016/j.brainres.2014.07.045

Hannila, S. S., Siddiq, M. M., Carmel, J. B., Hou, J., Chaudhry, N., Bradley, P. M., et al. (2013). Secretory leukocyte protease inhibitor reverses inhibition by CNS

\section{AUTHOR CONTRIBUTIONS}

BH: conceptualization, writing-original draft preparation and funding acquisition. $\mathrm{BH}$ and SJ: methodology and formal analysis. $\mathrm{BH}, \mathrm{SJ}$, and LK: writing-review and editing.

\section{FUNDING}

This study was supported by the Tyrolean Science Fund UNI0404/1920 (Amt der Tiroler Landesregierung).

\section{ACKNOWLEDGMENTS}

We thank Michaela Kind for excellent technical assistance.

myelin, promotes regeneration in the optic nerve and suppresses expression of the transforming growth factor $\beta$ signaling protein Smad2. J. Neurosci. 33, 5138-5151. doi: 10.1523/JNEUROSCI.5321-12.2013

Hausott, B., and Klimaschewski, L. (2019a). Promotion of peripheral nerve regeneration by stimulation of the extracellular signal-regulated kinase (ERK) pathway. Anat. Rec. 302, 1261-1267. doi: 10.1002/ar.24126

Hausott, B., and Klimaschewski, L. (2019b). Sprouty2-a novel therapeutic target in the nervous system? Mol. Neurobiol. 56, 3897-3903. doi: 10.1007/s12035-0181338-8

Hausott, B., Vallant, N., Auer, M., Yang, L., Dai, F., Brand-Saberi, B., et al. (2009). Sprouty2 down-regulation promotes axon growth by adult sensory neurons. Mol. Cell. Neurosci. 42, 328-340. doi: 10.1016/j.mcn.2009.08.005

Jones, D. M., Tucker, B. A., Rahimtula, M., and Mearow, K. M. (2003). The synergistic effects of NGF and IGF-1 on neurite growth in adult sensory neurons: convergence on the PI 3-kinase signaling pathway. J. Neurochem. 86, 1116-1128. doi: 10.1046/j.1471-4159.2003.01925.x

Kim, Y. J., Park, S. J., Choi, E. Y., Kim, S., Kwak, H. J., Yoo, B. C., et al. (2011). PTEN modulates miR-21 processing via RNA-regulatory protein RNH1. PLoS One 6:e28308. doi: 10.1371/journal.pone.0028308

Klimaschewski, L., Hausott, B., and Angelov, D. N. (2013). The pros and cons of growth factors and cytokines in peripheral axon regeneration. Int. Rev. Neurobiol. 108, 137-171. doi: 10.1016/b978-0-12-410499-0. 00006-x

Krichevsky, A. M., and Gabriely, G. (2009). miR-21: a small multi-faceted RNA. J. Cell. Mol. Med. 13, 39-53. doi: 10.1111/j.1582-4934.2008. 00556.x

Krishnan, A., Duraikannu, A., and Zochodne, D. W. (2016). Releasing 'brakes' to nerve regeneration: intrinsic molecular targets. Eur. J. Neurosci. 43, 297-308. doi: 10.1111/ejn.13018

Kwak, H. J., Kim, Y. J., Chun, K. R., Woo, Y. M., Park, S. J., Jeong, J. A., et al. (2011). Downregulation of Spry2 by miR-21 triggers malignancy in human gliomas. Oncogene 30, 2433-2442. doi: 10.1038/onc.2010.620

Leclere, P. G., Norman, E., Groutsi, F., Coffin, R., Mayer, U., Pizzey, J., et al. (2007). Impaired axonal regeneration by isolectin B4-binding dorsal root ganglion neurons in vitro. J. Neurosci. 27, 1190-1199. doi: 10.1523/JNEUROSCI.508906.2007

Lee, J. T., Steelman, L. S., Chappell, W. H., and McCubrey, J. A. (2008). Akt inactivates ERK causing decreased response to chemotherapeutic drugs in advanced CaP cells. Cell Cycle 7, 631-636. doi: 10.4161/cc.7.5.5416

Markus, A., Zhong, J., and Snider, W. D. (2002). Raf and akt mediate distinct aspects of sensory axon growth. Neuron 35, 65-76. doi: 10.1016/s08966273(02)00752-3

Marvaldi, L., Thongrong, S., Kozlowska, A., Irschick, R., Pritz, C. O., Bäumer, B., et al. (2015). Enhanced axon outgrowth and improved long-distance axon regeneration in sprouty2 deficient mice. Dev. Neurobiol. 75, 217-231. doi: 10.1002/dneu.22224

Mason, J. M., Morrison, D. J., Basson, M. A., and Licht, J. D. (2006). Sprouty proteins: multifaceted negative-feedback regulators of receptor 
tyrosine kinase signaling. Trends Cell Biol. 16, 45-54. doi: 10.1016/j.tcb.2005. 11.004

Masoumi-Moghaddam, S., Amini, A., and Morris, D. L. (2014). The developing story of Sprouty and cancer. Cancer Metastasis Rev. 33, 695-720. doi: 10.1007/s10555-014-9497-1

Ning, K., Drepper, C., Valori, C. F., Ahsan, M., Wyles, M., Higginbottom, A., et al. (2010). PTEN depletion rescues axonal growth defect and improves survival in SMN-deficient motor neurons. Hum. Mol. Genet. 19, 3159-3168. doi: $10.1093 / \mathrm{hmg} / \mathrm{ddq} 226$

Ning, L., Wang, C., Ding, X., Zhang, Y., Wang, X., and Yue, S. (2012). Functional interaction of TRPV4 channel protein with annexin A2 in DRG. Neurol. Res. 34, 685-693. doi: 10.1179/1743132812y.0000000065

Pan, C. W., Jin, X., Zhao, Y., Pan, Y. Q., Yang, J., Karnes, R. J., et al. (2017). AKT-phosphorylated FOXO1 suppresses ERK activation and chemoresistance by disrupting IQGAP1-MAPK interaction. EMBO J. 36, 995-1010. doi: 10.15252/embj.201695534

Patel, R., Gao, M., Ahmad, I., Fleming, J., Singh, L. B., Rai, T. S., et al. (2013). Sprouty2, PTEN, and PP2A interact to regulate prostate cancer progression. J. Clin. Invest. 123, 1157-1175. doi: 10.1172/jci 63672

Skouras, E., Ozsoy, U., Sarikcioglu, L., and Angelov, D. N. (2011). Intrinsic and therapeutic factors determining the recovery of motor function after peripheral nerve transection. Ann. Anat. 193, 286-303. doi: 10.1016/j.aanat.2011. 02.014

Strickland, I. T., Richards, L., Holmes, F. E., Wynick, D., Uney, J. B., and Wong, L. F. (2011). Axotomy-induced miR-21 promotes axon growth in adult dorsal root ganglion neurons. PLoS One 6:e23423. doi: 10.1371/journal.pone. 0023423

Sun, F., Park, K. K., Belin, S., Wang, D., Lu, T., Chen, G., et al. (2011). Sustained axon regeneration induced by co-deletion of PTEN and SOCS3. Nature 480, 372-375. doi: 10.1038/nature10594
Vazquez, F., and Sellers, W. R. (2000). The PTEN tumor suppressor protein: an antagonist of phosphoinositide 3-kinase signaling. Biochim. Biophys. Acta 1470, M21-M35. doi: 10.1016/s0304-419x(99)00032-3

Wang, X., Trotman, L. C., Koppie, T., Alimonti, A., Chen, Z., Gao, Z., et al. (2007). NEDD4-1 is a proto-oncogenic ubiquitin ligase for PTEN. Cell 128, 129-139. doi: 10.1016/j.cell.2006.11.039

Zhang, W., Lv, Y., Xue, Y., Wu, C., Yao, K., Zhang, C., et al. (2016). Co-expression modules of NF1, PTEN and sprouty enable distinction of adult diffuse gliomas according to pathway activities of receptor tyrosine kinases. Oncotarget 7, 59098-59114. doi: 10.18632/oncotarget.10359

Zhong, J. (2015). RAFting the rapids of axon regeneration signaling. Neural Regen. Res. 10, 341-343. doi: 10.4103/1673-5374.153670

Zhou, S., Shen, D., Wang, Y., Gong, L., Tang, X., Yu, B., et al. (2012). microRNA222 targeting PTEN promotes neurite outgrowth from adult dorsal root ganglion neurons following sciatic nerve transection. PLoS One 7:e44768. doi: 10.1371/journal.pone.0044768

Zimmermann, S., and Moelling, K. (1999). Phosphorylation and regulation of Raf by Akt (protein kinase B). Science 286, 1741-1744. doi: 10.1126/science.286. 5445.1741

Conflict of Interest: The authors declare that the research was conducted in the absence of any commercial or financial relationships that could be construed as a potential conflict of interest.

Copyright (c) 2020 Jamsuwan, Klimaschewski and Hausott. This is an open-access article distributed under the terms of the Creative Commons Attribution License (CC BY). The use, distribution or reproduction in other forums is permitted, provided the original author(s) and the copyright owner(s) are credited and that the original publication in this journal is cited, in accordance with accepted academic practice. No use, distribution or reproduction is permitted which does not comply with these terms. 